\title{
Clinical Outcomes of Montelukast Sodium in Children with Adenoid Hypertrophy
}

\author{
Jais Kumar* \\ Medical Qualification: MBBS and MD nephrology, Islamabad, Pakistan \\ *Corresponding author: Jais Kumar, Medical Qualification: MBBS and MD nephrology, Islamabad, Pakistan
}

ARTICLE INFO

Received: 蔧 April 06, 2020

Published: 慧 April 14, 2020

Citation: Jais Kumar. Clinical Outcomes of Montelukast Sodium in Children with Adenoid Hypertrophy. Biomed J Sci \& Tech Res 27(1)-2020. BJSTR. MS.ID.004438.

Keywords: Adenoid Hypertrophy; Montelukast Sodium; Opens Mouth Breathing

\begin{abstract}
Background: Generally the blockage of upper respiratory tract in children is seen with the hypertrophy of adenoids and tonsils. Normally for patients with adenoid hypertrophy $(\mathrm{AH})$, Adenoidectomy with or without Tonsillectomy is carried out, however it has its own genuine complications like haemorrhage and recurrence of adenoid tissue. Consequently, therapeutic treatment has increased extraordinary consideration rather than surgical procedure. The inflammatory process proposed for AH has prompted the utilization of anti-inflammatory drugs to treat this issue. The purpose of this study is to assess the impacts of Montelukast sodium in children with enlarged adenoids.
\end{abstract}

Materials and Methods: In this randomized, placebo treatment controlled trial, 60 children aged 4-12 years meeting inclusion criteria were isolated into two groups. The study group was treated with Montelukast sodium $5 \mathrm{mg}$ consistently for 3 months while the control group got placebo treatment for a similar timeframe. A questionnaire was filled by parents/ guardians of every child before and after the intervention to evaluate the severity of sleep discomfort, snoring and mouth breathing,

Results: Following 3 months of treatment, significant reduction in size of the adenoids was seen in $76 \%$ of study group compared with just $3 \%$ of control group getting placebo treatment.

Conclusion: Montelukast sodium is seen to be effective in the reduction of the size of adenoids and improvement in clinical manifestations and can be viewed as a viable option in contrast to surgical treatment in children with hypertrophy of adenoids.

\section{Introduction}

Adeno-tonsillar hypertrophy is the most widely recognized reason for upper respiratory tract blockage in youngsters with a pervasiveness of $2-3 \%$ [1]. The adenoid is a significant piece of Waldeyer's ring situated in nasopharyngeal region. As a result of its extraordinary area, in connection to the back choanae and Eustachian tube, it is regularly the site of beginning of numerous medical issues in youth. Adenoid is little in size at earliest stages yet increments in initial four years of life because of the improvement of immunity. Whenever left untreated, Adenoid Hypertrophy may prompt obstructive sleep apnea (snuffling, rest uneasiness, mouth breathing), ear issues, pulmonary hypertension, craniofacial peculiarities and inability to thrive [2]. In any case, the conclusion can be provocative in patients with manifestations of $\mathrm{AH}$. There are arrangements of proposals for the conclusion of AH however late examinations recommend that x-ray lateral neck and nasal endoscopy are the highest quality level examinations for AH [3]. Extended adenoids are expelled by adenoidectomy in pediatric age gathering however has a danger of post-operative complications like prompt and late hemorrhage (3-5\%) and reappearance of adenoid mass (10-20\%) of cases [1].

Significant expenses, nonattendance of child from school, risk of anesthesia and other surgical procedure related morbidities have additionally featured the need of medicinal treatment for adenoid hypertrophy. Levels of serum immunoglobulins have been seen to be lower in patients who have experienced adenotonsillectomy than in healthy control group [4]. Also, AH may 
create fundamental irritation in kids and grown-ups as shows by an expansion in C-reactive protein and this is connected with psychological and cardiovascular morbidity which diminishes following adeno-tonsillectomy. Leukotrienes are the main factor of inflammatory marker in the respiratory system. These mediators are engaged with the pathogenesis of childhood illness, for example, asthma and adenoid hypertrophy. Human cysteinyl-leukotriene receptor- 1 is markedly raised in the tonsillar tissue of youngsters with obstructive sleep apnea (OSA). Likewise, cysteinyl leukotriene receptor-1 which associates with leukotrienes and intervenes the provocative pathway was over communicated in adeno-tonsillar cells and tissues got from youngsters with $\mathrm{AH}$ [5].

Subsequently, leukotrienesanti-inflammatory agents with safe therapeutic profile may provide an effective interventional alternative to adeno tonsillectomy. Montelukast is FDA endorsed oral, cysteinyl-leukotriene receptor adversary which is powerful and very much endured preventive remedy in asthma and allergic rhinitis in children over 1 year. Additionally, Montelukast has not prompted resistance in long term studies [6,7]. The motivation behind this study was to evaluate the theory that Montelukast treatment may prompt improved nighttime symptoms, quality of life, and anatomical characteristics just as endoscopic and radiologic outcomes in youngsters with adenoid hypertrophy.

\section{Materials and Methods}

This Randomized Controlled Preliminary was performed from April 2018 to March 2019 in the Otorhinolaryngology Clinic of Dr Akbar Niazi teaching hospital, Islamabad. 60 children meeting the inclusion criteria were enrolled. Analysis was set up based on clinical assessment, lateral neck radiograph, Flexible nasal endoscopy and x-ray post nasal space (soft tissue neck lateral view).

\section{Inclusion Criteria}

- $\quad$ Children more than 4 and less than 12 years old with snoring

- $\quad$ Grade 3 or more noteworthy nasopharynx deterrent on endoscopic assessment

- $\quad 50 \%$ or more in Adenoidal/Nasopharyngeal proportion in radiographic examinations.

\section{Exclusion Criteria}

- $\quad$ Obesity characterized as BMI for age percentile $>1.645$ $(95 \%)$

- $\quad$ Genetic irregularities

- $\quad$ Previous or current utilization of Montelukast

- $\quad$ Acute infection of upper respiratory tract

- Any anti-microbials or corticosteroids use a month prior to think about Children with previous history of adenotonsillectomy
Children were examined by a particular otorhinolaryngologist and were allotted to study or control group $(n=30)$. The study group got Montelukast 4 and 5mg every day for children less than 6 and more than 6 years old separately. While placebo treatment tablets with a similar measurements were recommended for the control group. All guardians were instructed to give the tablets at sleep time. Second nasal endoscopy alongside X-ray neck was performed following 3 months treatment course.

\section{Lateral Neck Radiography study:}

To evaluate the patency of airway, lateral neck radiographs were performed utilizing the standard system in the Radiology department at Dr. Akbar Niazi Teaching Hospital Islamabad. Patient was told to inhale through the nose with the mouth shut and neck broadened. Adenoidal/nasopharyngeal proportion was estimated utilizing the technique depicted by Fujioka and colleagues $[8,9]$. X-ray lateral neck was performed before the start of study and repeated following 3 months treatment course. Flexible nasal endoscopy was performed to acquire a full choanal picture. Topical anesthesia and vasoconstrictors were introduced in all patients experiencing nasal endoscopy. The measurement of obstruction was classified utilizing Parikh technique, which depends on anatomical relationship [10,11].

Evaluation $0=$ None, Evaluation $1=$ Torus Tubarius, Evaluation 2 = Torus Tubarius and Vomer, Evaluation 3 = Vomer and soft palate. Flexible nasal endoscopy was performed toward the beginning and end of study. The primary outcome was mouth breathing and restlessness. Secondary result measures were the adenoid size based on naso-endoscopy and lateral neck soft tissue radiograph. Based on the severity of symptom, a score $(0-3)$ was structured which is given as pursues; 0 - Never existed, 1 - Intermittent manifestations, 2 - Present all things considered occasions 3 - Constantly present. All numerical data was subjected to statistical analysis with Mann whitney Test. $\mathrm{P}<0.05$ was considered significant.

\section{Results}

Sixty children meeting the inclusion criteria were considered. No withdrawals or any reactions were seen in any of the patients. In our study, primary symptoms were open mouth breathing, snoring and restlessness. As indicated by the Mann Whitney Test, no distinction was seen in snoring between the two groups $(\mathrm{P}=0.111) .3$ were the mean score in each group. However, a significant difference was seen between the two groups after treatment $(\mathrm{P}<0.007)$ (Figures 1-3). Regarding restlessness, no critical difference between the two groups was seen toward the start of study ( $\mathrm{P}=0.408)$. However statistically significant difference observed after treatment $(\mathrm{P}<0.0001)$. For the indication of mouth breathing, the mean score of the treatment group was higher toward the beginning of study, yet the little distinction was not measurably significant. Methods for nasal endoscopy and lateral neck radiography were additionally used to ponder the patient's side effects toward the beginning 
and end of treatment course. The mean score between the two groups uncovered an important distinction before the treatment
$(P=0.03)$, yet a progressively significant difference was seen after the treatment course $(\mathrm{P}<0.0001)$.

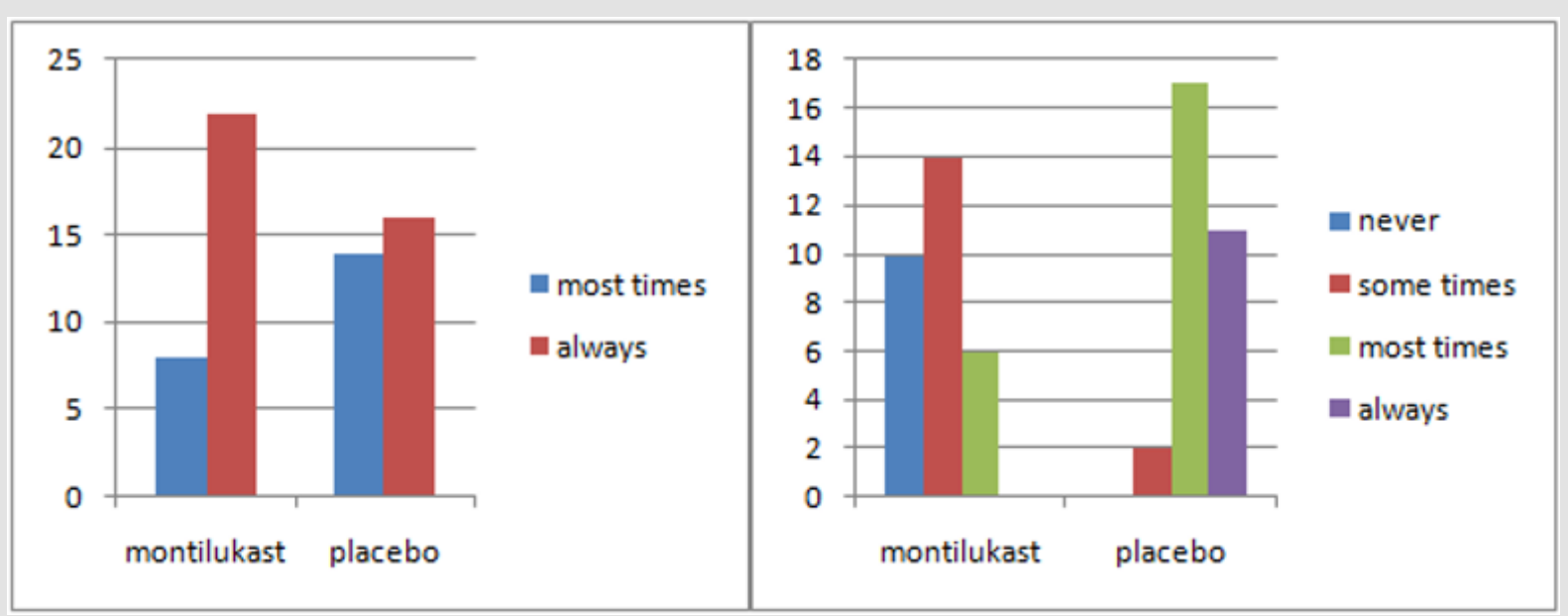

Figure 1: Snoring scores before and after treatment.

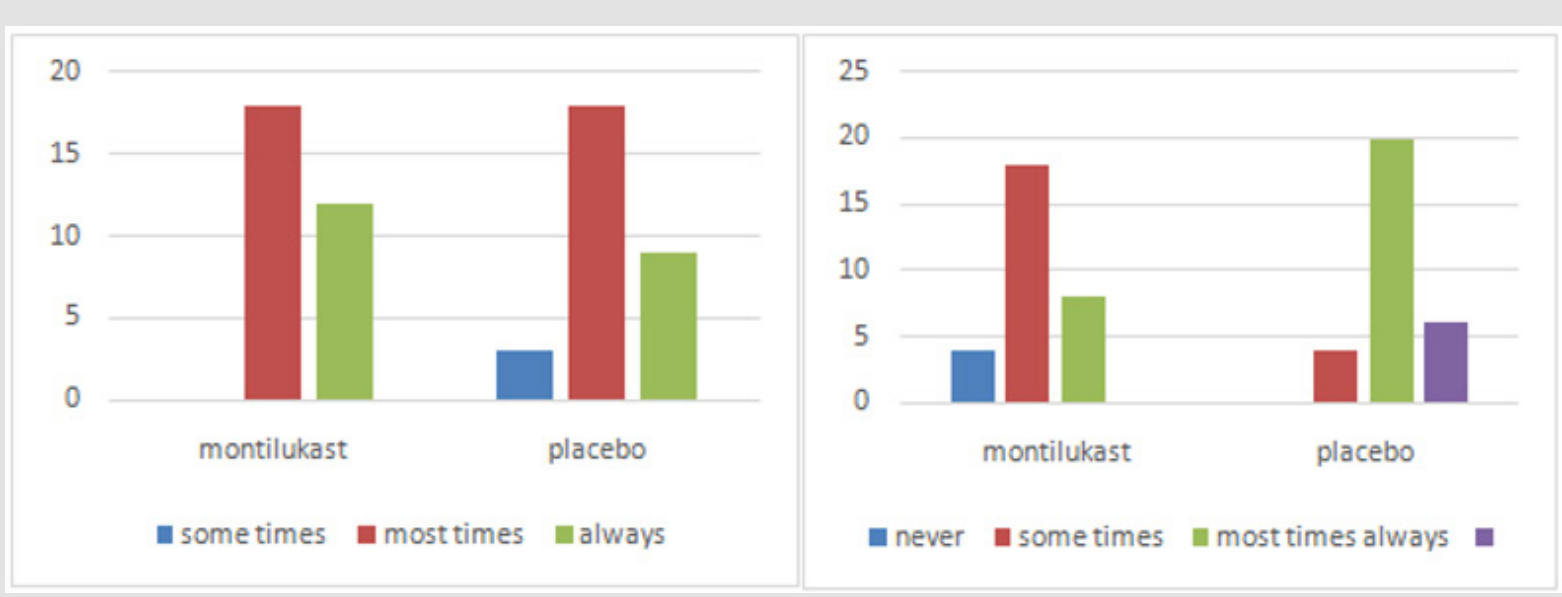

Figure 2: Before and after treatment of Sleep discomfort score.
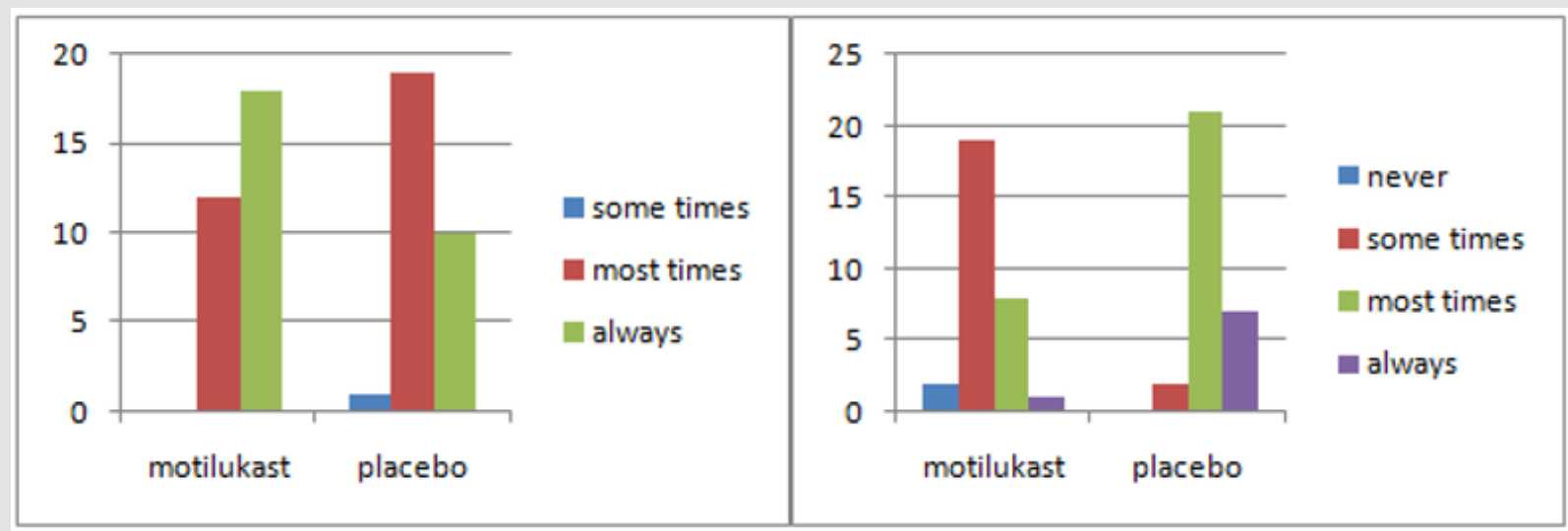

Figure 3: Before and after treatment of Mouth breathing score.

In radiographic examinations, a marked decrease of $\geq 25 \%$ was seen in the size of adenoids after treatment. The response rate was $76 \%$ in the treated group and 3.3\%inplacebo group. $\mathrm{P}<0.0001$ was considered measurably significant. Correlation of these two clinical tests in connection to the clinical side effects was finished utilizing
Cohen's Kappa coefficient which is a proportion of understanding. This uncovered an estimation of 0.55 for radiography and 0.8 for naso-endoscopy. Subsequently, naso-endoscopy had a solid relationship with the patient's manifestations and his/her general condition (Tables 1 \& 2). 
Table 1: statistic attributes of montelukast sodium and placebo treatment.

\begin{tabular}{|c|c|c|c|}
\hline & & Montelukast $\mathbf{n = ( 3 0 )}$ & Placebo $\mathbf{n = ( 3 0 )}$ \\
\hline \multirow{2}{*}{ Sex } & Male & 19 & 15 \\
\cline { 2 - 4 } & Female & 11 & 15 \\
\hline \multirow{2}{*}{ Age (yrs.) } & & $6.9 \pm 2.33$ & $6.83 \pm 2.36$ \\
\hline
\end{tabular}

Table 2.

\begin{tabular}{|c|c|c|c|c|}
\hline & \multicolumn{2}{|c|}{ Montelukast } & \multicolumn{2}{c|}{ Placebo } \\
\hline & Before & After & Before & After \\
\hline Endoscopy (score) & $3.7667 \pm 0.43018$ & $2.3667 \pm 0.76489$ & $3.5667 \pm 50401$ & $3.4333 \pm 0.56832$ \\
\hline Radiography (\%) & $\pm 87.23 \pm 8.97$ & $\pm 51.33 \pm 5.91$ & $81.16 \pm 7.27$ & $77.83 \pm 9.06$ \\
\hline
\end{tabular}

\section{Discussion}

Adenoids are lympho-epithelial organs confined to the top of nasopharynx region. It typically reaches at their most extreme size between 3 to 6 years old and then regresses. Adenoid hypertrophy is frequently pursues upper respiratory tract infection and is a typical illness of childhood. Chronic infections are the most widely recognized signs of physiological and pathological changes in the adenoids. It might prompt nasal blockage, mouth breathing, restlessness, contaminations in the ear and sinuses and diminished personal satisfaction. Normally adenoid tissue relapses with the progression of time if the infection doesn't occur. Adenoid tissue may re grow even after adenoidectomy because of chronic hypersensitive responses or infections [12]. Because of medical procedure related intricacies like massive bleeding, velopharyngeal insufficiency and risks of anesthesia, alternative treatment methodologies have grown over time. Our study exhibited that Montelukast sodium's an oral, chewable tablet given to the children with $\mathrm{AH}$ for 3 months has successfully diminished the seriousness of breathing difficulty, snoring and mouth breathing along with decrease in the size of adenoid tissue. Moreover, this treatment was all around endured by the patients with no side effects.

Notable decrease in adenoid size was affirmed through lateral neck soft tissue x-rayand naso-endoscopy. Regardless of the troubles in performing nasal endoscopy, it has a solid connection with the clinical symptoms [11]. Shokouhi et al. [13] also observed a significant reduction in the size of adenoids in a group receiving Montelukast which supports our study. In a study by Goldbart et al. [14], Montelukast sodium was utilized in the treatment of Obstructive Sleep Apnea in fourty children between 4 to 12 years. 20 children received $4 \mathrm{mg}$ chewable tablets of Montelukast sodium for children less than 6 years old and $5 \mathrm{mg}$ for those greater than 6years while the other twenty got placebo treatment both for 3 months. A noteworthy improvement of more than $50 \%$ was seen in polysomno graphic parameters and the Adenoidal Nasopharyngeal proportion in radiography decreased from $81 \%$ to $57 \%$ (Cohen kappa coefficient 0.55 ). The difference in the outcomes may be because of variety in the measurements of medication utilized $4 \mathrm{mg}$ for children less than 6 years and $5 \mathrm{mg}$ for more than 6years of age in our study or the distinction in the severity of manifestations towards the start of study. We found moderate to severe symptoms in our study while in the previously mentioned study; the manifestations were mild tomoderate. Tuhanioğlu B and Erkan SO [15] additionally discovered relapse in the adenoid size in the group have taken Montelukast sodium. Generally, montelukast can be recommended as a substitute to surgical procedure to prevent post-operative complications.

\section{Conclusion}

A notable decrease observed in the size of adenoid tissue along with improvement in the clinical symptoms with Montelukast sodium treatment. So it is considered as an effective substitute to surgical procedure in children with enlarged adenoids.

\section{References}

1. Liming BJ, Ryan M, Mack D, Ahmad I, Camacho M (2019) Montelukast and Nasal Corticosteroids to Treat Pediatric Obstructive Sleep Apnea: A Systematic Review and Meta-analysis. Otolaryngology-Head and Neck Surgery 160(4): 594-602.

2. Yan Y, Song Y, Liu Y, Su J, Cui L, et al. (2019) Early Stage Impacts of Adenoidectomy With/Without Tonsillectomy on Immune Functions of Children aged $<3$ Years. Pediatric Allergy Immunology and Pulmonology 32(1): 18-22.

3. Sharifkashani S, Dabirmoghaddam P, Kheirkhah M, Hosseinzadehnik R (2015) A new clinical scoring system for adenoid hypertrophy in children. Iranian journal of otorhinolaryngology 27(78): 55.

4. Babakurban ST, Aydın E (2016) Adenoidectomy: Current approaches and review of the literature. Kulak Burun BogazIhtis Derg 26(3): 181190.

5. Kheirandish Gozal L, Bandla HP, Gozal D (2016) Montelukast for children with obstructive sleep apnea: Results of a double-blind, randomized, placebo-controlled trial. Annals of the American Thoracic Society 13(10): 1736-1741.

6. cadding G (2010) Non-surgical treatment of adenoidal hypertrophy: The role of treating Ig E-mediated inflammation. Pediatric Allergy and Immunology 21(8): 1095-1106.

7. Bozkurt MK, Tulek B, Bozkurt B, Akyürek N, Mehmet ÖZ, et al. (2014) Comparison of the efficacy of prednisolone, montelukast, and omalizumab in an experimental allergic rhinitis model. Turkish journal of medical sciences 44(3): 439-447.

8. Cohen D, Konak S (1985) The evaluation of radiographs of the nasopharynx. Clin Otolaryngol Allied Sci 10(2): 73-78. 
9. Fujioka M, Young LW, Girdany BR (1979) Radiographic evaluation of adenoidal size in children: adenoidal-nasopharyngeal ratio. AJR Am Roentgenol 133(3): 401-404.

10. Parikh SR, Coronel M, Lee JJ, Brown SM (2006) Validation of a new grading system for endoscopic examination of adenoid hypertrophy. Otolaryngol Head Neck Surg 135(5): 684-687.

11. Caylakli F, Hizal E, Yilmaz I, Yilmazer C (2009) Correlation between adenoid-nasopharynx ratio and endoscopic examination of adenoid hypertrophy: A blind, prospective clinical study. Int J Pediatr Otorhinolaryngol 73(11): 1532-1535.

12. Dixit Y, Tripathi PS (2016) Community level evaluation of adenoid hypertrophy on the basis of symptom scoring and its X-ray correlation. J Family Med Prim Care 5: 789-791.

ISSN: 2574-1241

DOI: $10.26717 /$ BJSTR.2020.27.004438

Stefan Bittmann. Biomed J Sci \& Tech Res

(C) This work is licensed under Creative

Submission Link: https://biomedres.us/submit-manuscript.php
13. Shokouhi F, Jahromi AM, Majidi MR, Salehi M (2015) Montelukast in adenoid hypertrophy: Its effect on size and symptoms. Iranian Journal of Otorhinolaryngology 27: 443-448.

14. Goldbart AD, Greenberg Dotan S, Tal A (2012) Montelukast for children with obstructive sleep apnea: a double-blind, placebo-controlled study. Pediatrics 130(3): e575-e580.

15. Tuhanıoğlu B, Erkan SO (2017) Evaluation of the effects of montelukast mometasonefuroate and combined therapyon adenoid size: A randomized prospective clinical trial with objective data. Turk J Med Sci. 47(6): 1736-1743.

$\begin{array}{ll}\text { BIOMEDICAL } & \text { Assets of Publishing with us } \\ \text { RESEARCHES } & \text { - Global archiving of articles } \\ \text { - Immediate, unrestricted online access }\end{array}$

